\title{
Phosphorylation of Zipcode Binding Protein 1 Is Required for Brain-Derived Neurotrophic Factor Signaling of Local $\beta$-Actin Synthesis and Growth Cone Turning
}

\author{
Yukio Sasaki, ${ }^{1,3}$ Kristy Welshhans, ${ }^{1}$ Zhexing Wen, ${ }^{1,4}$ Jiaqi Yao, ${ }^{4}$ Mei Xu, ${ }^{1}$ Yoshio Goshima, ${ }^{3}$ James Q. Zheng, ${ }^{1,4}$ \\ and Gary J. Bassell ${ }^{1,2}$ \\ Departments of ${ }^{1}$ Cell Biology and ${ }^{2}$ Neurology, Emory University School of Medicine, Atlanta, Georgia 30322, ${ }^{3}$ Department of Molecular Pharmacology and \\ Neurobiology, Yokohama City University Graduate School of Medicine, Yokohama 236-0004, Japan, and ${ }^{4}$ Department of Neuroscience and Cell Biology, \\ University of Medicine and Dentistry of New Jersey, Robert Wood Johnson Medical School, Piscataway, New Jersey 08854
}

The localization of specific mRNAs and their local translation in growth cones of developing axons has been shown to play an important mechanism to regulate growth cone turning responses to attractive or repulsive cues. However, the mechanism whereby local translation and growth cone turning may be controlled by specific mRNA-binding proteins is unknown. Here we demonstrate that brain-derived neurotrophic factor (BDNF) signals the Src-dependent phosphorylation of the $\beta$-actin mRNA zipcode binding protein 1 (ZBP1), which is necessary for $\beta$-actin synthesis and growth cone turning. We raised a phospho-specific ZBP1 antibody to Tyr396, which is a Src phosphorylation site, and immunofluorescence revealed BDNF-induced phosphorylation of ZBP1 within growth cones. The BDNF-induced increase in fluorescent signal of a green fluorescent protein translation reporter with the $3^{\prime}$ untranslated region of $\beta$-actin was attenuated with the Src family kinase-specific inhibitor PP2 [4-amino-5-(4-chlorophenyl)-7-(t-butyl)pyrazolo[3,4- $d$ ]pyrimidine]. Furthermore, a nonphosphorylatable mutant, ZBP1 Y396F, suppressed the BDNF-induced and protein synthesis-dependent increase in $\beta$-actin localization in growth cones. Last, the ZBP1 Y396F mutant blocked BDNF-induced attractive growth cone turning. These results indicate that phosphorylation of ZBP1 at Tyr396 within growth cones has a critical role to regulate local protein synthesis and growth cone turning. Our findings provide new insight into how the regulated phosphorylation of mRNA-binding proteins influences local translation underlying growth cone motility and axon guidance.

\section{Introduction}

The localization of specific mRNAs to peripheral sites within polarized cells provides a conserved biological mechanism to regulate protein sorting (Martin and Ephrussi, 2009). There is a growing appreciation that the molecular mechanisms for mRNA localization and local translation involve interactions between specific mRNA-binding proteins and their target sequences, often in the $3^{\prime}$ untranslated region (UTR), but it remains unclear how such interactions are regulated by physiological signals. In neurons, the regulation of local protein synthesis in response to neurotrophins, axon guidance factors, and neurotransmitters is a fundamental mechanism to regulate axon guidance and synaptic plasticity (Steward and Schuman, 2001; Piper and Holt, 2004; Sutton and Schuman, 2006; Bramham and Wells, 2007; Lin and Holt, 2007), but the mechanisms whereby specific mRNAbinding proteins may regulate these events are unclear (Kiebler

Received Jan. 29, 2010; revised Feb. 21, 2010; accepted May 10, 2010.

This work was supported by National Institutes of Health Grants R01 HD46368-08 and HD055835 (G.J.B.), R01 NS036241 (J.Q.Z.), and 5F32NS064727 (K.W.) and by the Special Coordination Funds for Promoting Science and Technology (Y.G.). We thank Lei Xing for helpful discussions and technical assistance. We thank Stefan Huttelmaier and Robert Singer for providing the ZBP1 (Y396F) mutant construct and helpful discussions that initiated this study

Correspondence should be addressed to Gary J. Bassell, Department of Cell Biology, Emory University, 615 Michael Street, Atlanta, GA 30322. E-mail: gbassel@emory.edu.

DOI:10.1523/JNEUROSCI.0499-10.2010

Copyright $\odot 2010$ the authors $\quad 0270-6474 / 10 / 309349-10 \$ 15.00 / 0$ and Bassell, 2006). A few mRNA-binding proteins have been implicated to couple mRNA transport to local translation in neurons (Bassell and Kelic, 2004; Kiebler and Bassell, 2006). These include zipcode binding protein (ZBP1), cytoplasmic polyadenylation element binding protein (CPEB), and fragile X mental retardation protein (FMRP). In each case, these proteins serve a dual function: they act as both a translational repressor and an mRNA transport factor. An emerging hypothesis is that such mRNA-binding proteins act as key molecular intermediates to mediate the switch from translational repression in response to physiological signals.

ZBP1 binds to a 54 nt zipcode sequence located in the $3^{\prime}$ UTR of $\beta$-actin mRNA; this interaction is necessary for mRNA localization to the leading edge of fibroblasts (Ross et al., 1997; Farina et al., 2003) or the growth cone of a developing neuron (Zhang et al., 2001). Antisense oligonucleotides to the zipcode sequence impair localization of $\beta$-actin mRNA to growth cones in response to stimulation by neurotrophin-3 (Zhang et al., 2001). More recently, $\beta$-actin mRNA and Xenopus ZBP1 (VgRBP, for Vg1 RNAbinding protein) were concentrated and colocalized on the near side of growth cones turning toward sources of brain-derived neurotrophic factor (BDNF) (Yao et al., 2006). Furthermore, BDNF and netrin-1 both induced local translation of $\beta$-actin mRNA in growth cones (Leung et al., 2006; Yao et al., 2006). Disruption of the interaction between ZBP1 and $\beta$-actin mRNA 
using acute application of antisense morpholino oligonucleotides (AMOs) against the zipcode sequence suppressed attractive growth cone turning toward a focal source of BDNF (Yao et al., 2006). Similarly, AMOs to block the synthesis of new actin protein also impaired attractive turning of growth cones toward a netrin gradient (Leung et al., 2006). Together, these findings demonstrate that localization of $\beta$-actin mRNA and local $\beta$-actin synthesis is necessary for growth cone turning and suggest a possible function for ZBP1. However, the requirement of ZBP1 and whether it provides a critical convergent point for signaling of translation-mediated growth cone turning has not been demonstrated.

ZBP1 has been shown to repress the global translation of $\beta$-actin mRNA in whole cells using a luciferase assay (Hüttelmaier et al., 2005). In addition, phosphorylation of ZBP1 at Tyr396 by Src results in a marked reduction in the affinity of ZBP1 for $\beta$-actin mRNA, suggesting a possible local mechanism to activate translation (Dahm and Kiebler, 2005; Hüttelmaier et al., 2005). Here we have addressed three key questions. Is ZBP1 phosphorylation regulated by physiological signals? Where in the cell does ZBP1 phosphorylation occur? Is ZBP1 phosphorylation necessary for local translation and cell motility? Our findings reveal that tyrosine phosphorylation of ZBP1 by Src family kinases (SFKs) is involved in local translation of $\beta$-actin in growth cones and growth cone turning in response to BDNF signaling. This study provides new insight into how mRNA-binding proteins regulate growth cone turning via local protein synthesis in growth cones.

\section{Materials and Methods}

Antibodies and Western blot. Polyclonal anti-ZBP1 antibody was produced by immunizing guinea pigs with a synthetic peptide corresponding to residues 162-175 (CGPENGRRGGFGSRG, the first Cys is for conjugation) within a hinge region between two RRM and four $\mathrm{KH}$ domains of rat ZBP1 as described previously (Santangelo et al., 2009). This region is conserved completely among mouse, rat, and human ZBP1/insulin like growth factor-II (IGF-II) mRNA binding protein (IMP1) but not IMP2 or IMP3 homologs. ZBP1 is also known as IMP1, one of three homologs described for their binding to a sequence in the $5^{\prime}$ UTR of Igf2 mRNA that is involved in translational regulation (Nielsen et al., 1999). IMP3 is the ortholog of VgRBP, the Xenopus protein that interacts with $\beta$-actin mRNA (Leung et al., 2006). Our anti-ZBP1 antibody does not recognize mouse IMP2 or IMP3 by Western blotting (supplemental Fig. S1, available at www.jneurosci.org as supplemental material).

Polyclonal anti-phospho-ZBP1 antibody was produced by immunizing rabbits with a phospho-synthetic peptide corresponding to residues 390-404 (CVTGAAPpYGSFMQAPE, pY is phospho-tyrosine at 396, and the first Cys is for conjugation) within a hinge region between the second and third KH domains of rat ZBP1 (Hüttelmaier et al., 2005) by Abgent. The anti-phospho-ZBP1 antibody does not detect phosphorylated IMP2 or IMP3 by Fyn expression analyzed using Western blotting (supplemental Fig. S1, available at www.jneurosci.org as supplemental material). Both antibodies were affinity purified using an antigen peptide-conjugated column. For Western blot analysis of ZBP1 phosphorylation, HEK293T cells were transfected with green fluorescent protein (GFP)-fused ZBP1 with or without myc-tagged Fyn (Sasaki et al., 2002). After $36 \mathrm{~h}$, the cells were harvested by scrapers in lysis buffer [20 mm Tris $\mathrm{HCl}$, pH 7.4, $100 \mathrm{~mm} \mathrm{NaCl}, 1 \%$ Triton X-100, 2 mм EDTA, 1 mм $\mathrm{Na}_{3} \mathrm{VO}_{4}$ (Sigma), $10 \mu \mathrm{m}$ phenylarsine oxide (Sigma), and protease inhibitors (Complete Mini; Roche Applied Science)].

To analyze phosphorylation of ZBP1 in neurons, dissociated rat neuronal cultures were prepared from cortex at embryonic day 18 (E18) and cultured in Neurobasal medium plus B27 supplement, using the Banker method (Goslin et al., 1998). After 3 d, the dissociated cultures were starved by removing B27 from the medium for $2 \mathrm{~h}$, stimulated by 100 $\mathrm{ng} / \mathrm{ml}$ BDNF (Peprotech), and then harvested by scrapers in the lysis buffer. Protein extracts were resolved by $8 \%$ SDS-PAGE, and fractionated proteins were transferred to a polyvinylidene difluoride membrane (Bio-Rad) at $4^{\circ} \mathrm{C}$ overnight. The anti-phospho-ZBP1, the anti-ZBP1, mouse anti-GFP (JL-8; Clontech), and anti-Myc (9E10; Sigma) antibodies were used as primary antibodies at 1:2000, 1:3000, 1:5000, and 1:5000, respectively. The membrane was washed and incubated with peroxidaseconjugated goat anti-rabbit, mouse (both from GE Healthcare), and guinea pig (Jackson ImmunoResearch) IgGs. The membrane was exposed to x-ray film (Eastman Kodak), using ECL detection reagents (GE Healthcare).

Neuron balls and dissociated cultures of transfected cortical neurons. Rat cortical neurons were dissected from E18 embryos. After trypsinization of cells, plasmids were transfected using Amaxa nucleofection kit (Lonza). Transfected cells were seeded at 10,000 cells $/ \mathrm{cm}^{2}$ and then cultured for $3 \mathrm{~d}$ as dissociated culture. Relative expression level of mCherry ZBP1 to endogenous ZBP1 was 2:1 in the dissociated cortical neurons (supplemental Fig. S2, available at www.jneurosci.org as supplemental material). In the case of neuron ball cultures, drops of the cell suspension in Neurobasal media, including GlutaMax and B27 supplement (all from Invitrogen) and $10 \%$ horse serum, were placed on the top cover (inside surface) of $100 \mathrm{~mm}$ dishes. One drop contains 10,000 cells/10 $\mu$ l. The cover was inverted onto the bottom half of the $100 \mathrm{~mm}$ dish that contains water to maintain humidity. After $3 \mathrm{~d}$ of incubation in $\mathrm{CO}_{2}$ incubator as hanging drop cultures, the neurons will form a ball in each drop. "Neuron balls" were then manually transferred and placed individually on poly-L-lysine-coated dishes containing Neurobasal media plus B27 at 5 mm intervals. After $1 \mathrm{~d}$, cytosine $\beta$-D-arabinofuranoside hydrochloride ( $3 \mu \mathrm{M}$; Sigma) was added to the media to kill dividing cells. Neuron balls extended neurites up to $1 \mathrm{~mm}$ for $3 \mathrm{~d}$, and most of the neurites were axons (supplemental Fig. S6, available at www.jneurosci.org as supplemental material).

Immunofluorescence and digital imaging. For immunofluorescence (IF), dissociated and neuron ball cultures were starved by removing B27 from the medium for $2 \mathrm{~h}$, stimulated by $100 \mathrm{ng} / \mathrm{ml}$ BDNF, and then fixed at specified times with $4 \%$ paraformaldehyde in PBS. In some experiments, anisomycin $(20 \mu \mathrm{g} / \mathrm{ml})$ was applied $30 \mathrm{~min}$ before BDNF stimulation. IF was performed as described (Eom et al., 2003; Tiruchinapalli et al., 2003). Briefly, primary antibodies were incubated for $1 \mathrm{~h}$, and then cyanine 3 (Cy3) and Cy5 fluorochrome-conjugated anti-rabbit, mouse, and guinea pig antibodies were incubated as secondary antibodies (1:500, respectively; Jackson ImmunoResearch). The following primary antibodies were used: rabbit anti-phospho-ZBP1 (1:1000), guinea pig antiZBP1 (1:300), mouse anti- $\beta$-actin (AC-15, 1:1000; Abcam), and rabbit anti-FLAG (1:1000; Sigma) antibodies. Alexa 488-conjugated phalloidin was used to detect filamentous actin (F-actin) (1:1000; Invitrogen). IF images were visualized using a $60 \times$ Plan-Neofluar objective, $120 \mathrm{~W}$ metal halide lamp (X-cite 120 PC; EXFO Life Sciences), and HiQ bandpass filters (Chroma Technology) on an Eclipse TE-300 (Nikon) inverted microscope, as described previously (Eom et al., 2003). Images were captured with a cooled CCD camera (Quantix; Photometrics) using IP Lab software (Scanalytics). Mean IF intensities for phospho- and total ZBP1 and $\beta$-actin in growth cones were analyzed within individual growth cones as regions of interest (ROIs). Total IF intensity of the ROI was quantified and then divided by area of the ROI to normalize for differences in growth cone area. Growth cones were defined as phalloidin-stained or GFP-positive areas at the tip of axons. The resulting total intensity/area was subtracted by a mean of two regions (background/area) near individual growth cones and then was defined as a normalized IF intensity in an individual growth cone. Each experiment was repeated a minimum of three times, imaging at least 10 growth cones, using a paired Student's $t$ test or ANOVA.

Fluorescence in situ hybridization. Three digoxigenin (DIG)-labeled oligonucleotide probes were used to detect rat $\beta$-actin mRNA. The oligonucleotide sequences were as follows: $5^{\prime}$-TGTTCAATGGGGTACTTCAGGGTCAGGATACCTCTCTTGCTCTGGGCCTCGTC3', 5' -TGAGGAAAGTAGGGTTGATGAGGCCAGCTTGGCCAGGTGTCAGGGAGATACCTTC- ${ }^{\prime}$ ' and $5^{\prime}$-AATGCCTGTGGTACGACCAGAGGCATACAGGGACAGCACAGCCTGGATGGCTAC-3'. Fluorescence in situ hybridization (FISH) was performed as described previously 
A

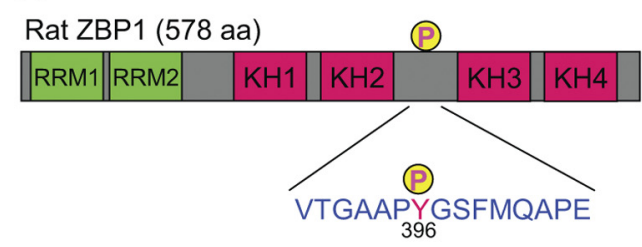

\section{B}

Rat ZBP1
Mouse $\mathrm{ZBP} 1$
Human $\mathrm{ZBP} 1$
Chick $\mathrm{ZBP1}$
Mouse IMP2
Mouse IMP3
Xen. VgRBP

\section{C}

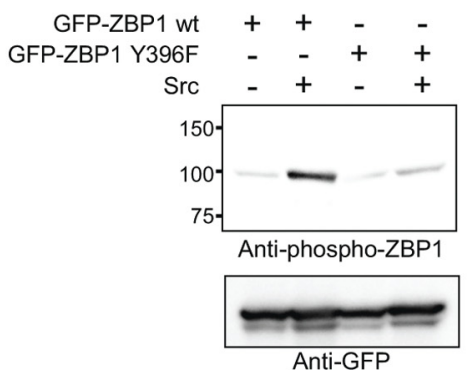

390-404 VTGAAPYGSFMQAPE

390-404 VTGAAPYSSEMQAPE 390-404 VTGAAPYSSEMQAPE 390-404 VSGAAPYSSFMP-PE

382-396 VPPSPPYHPEATHSG 391-404 STLTPPYPQEEQS-E 403-417 PTSSTSYPPEGQQPE
D

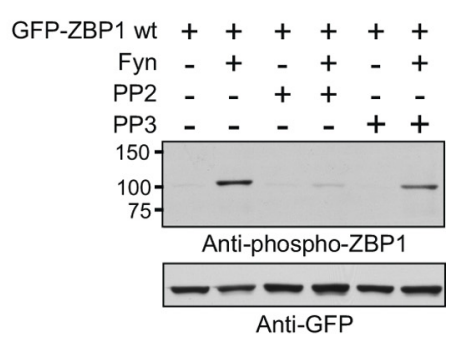

E

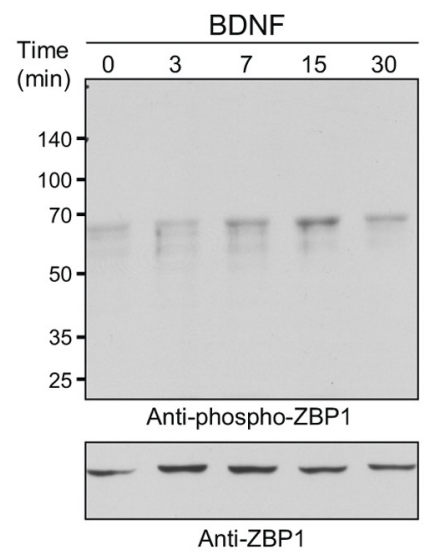

Figure 1. Phosphorylation of ZBP1 by BDNF in rat cortical neurons. $A$, Schematic diagram of rat ZBP1 domain structure and amino acid sequence of the linker region between the KH2 and KH3 domains. Phosphorylation site at Tyr396 is indicated. $\boldsymbol{B}$, Sequence alignment of ZBP1/IMP1 in four species and homologs (IMP2, IMP3, and VgRBP). Although the phosphorylated tyrosine position is conserved among all members of the IMP family, the sequence surrounding the tyrosine is conserved only in ZBP1. Red residues, Phosphorylatable tyrosine; blue residues, conserved residues between species or within ZBP1 homologs. Xen., Xenopus. C, Specificity of anti-phospho-ZBP1 antibody. Indicated plasmids were transfected into HEK293T cells and analyzed with immunoblotting. Overexpression of constitutively active Src tyrosine kinase increased the immunoreactive band corresponding to GFP-ZBP1, whereas no signal against the Y396F mutant was detected. D, The effect of SFK inhibitor. GFP-ZBP1 and Fyn, a neuronal SFK, were transfected into HEK293T cells, and the cells were treated with an SFK inhibitor. The SFK-specific inhibitor PP2, but not PP3 (an inactive derivative of PP2), inhibited the increase in ZBP1 phosphorylation induced by Fyn. $\boldsymbol{E}$, Time course of Tyr396 phosphorylation of endogenous ZBP1 in rat cortical neurons after stimulation by BDNF. Stimulation of cortical neurons with BDNF resulted in an increase in Tyr396 phosphorylation of endogenous ZBP1, which reached its maximum at 15 min and decreased thereafter.

with some modifications (Bassell et al., 1998; Zhang et al., 2001). Briefly, the fixed neurons were rinsed in PBS/ $0.2 \mathrm{mM} \mathrm{MgCl}_{2}$ and equilibrated in $1 \times$ SSC. They were then transferred to $40 \%$ formamide $/ 1 \times$ SSC before preincubation in hybridization buffer $(25 \%$ dextran sulfate, $2 \times$ SSC, $0.4 \%$ bovine serum albumin, $20 \mathrm{~mm}$ ribonucleoside vanadyl complex, and $10 \mathrm{~mm}$ sodium phosphate buffer, $\mathrm{pH}$ 7.0). The neurons were hybridized with DIG-labeled oligonucleotide probes in hybridization buffer at $37^{\circ} \mathrm{C}$ overnight. After stringent washes with $1 \times$ SSC, they were incubated with Cy5-labeled mouse anti-DIG antibody to visualize hybridization signals. In addition, the neurons were also incubated with rabbit antiFLAG antibody to label the flag-mCherry-ZBP1 transfected neurons for $2 \mathrm{~h}$, followed by Cy3-labeled anti-rabbit antibodies for $1 \mathrm{~h}$ (Jackson ImmunoResearch). For negative controls, DIG-labeled scrambled oligonucleotide probe was used.

Analysis of local protein synthesis using fluorescent reporters. Local protein synthesis was measured by calculating the fluorescence ratio of two reporters [enhanced GFP (EGFP) and mCherry], with and without full length of $3^{\prime}$ UTR of rat $\beta$-actin sequences. EGFP and mCherry cDNA were fused to a dual palmitoylation sequence from GAP-43 at the $\mathrm{N}$ termini and destabilized sequence from the d2EGFP vector (Clontech) to $\mathrm{C}$ termini. Among the resulting two vectors (gd2G and gd2R), gd2 $\mathrm{G}$ was fused to 3' UTR of rat $\beta$-actin sequences. The GFP reporter (gd2G) with or without the $3^{\prime}$ UTR was cotransfected with the mCherry reporter gd2R lacking the 3' UTR into cortical neurons using Amaxa nucleofection kit. After transfection, neuron balls were prepared by incubating as hanging drop cultures for $3 \mathrm{~d}$. Neuron balls were then put on glass-bottom dishes for live imaging (delta T dish; Bioptechs) and cultured for an additional $3 \mathrm{~d}$. Before live cell imaging, the medium was changed to phenol red-free and HEPES-buffered Neurobasal medium without B27, and the culture was incubated for $2 \mathrm{~h}$. Just before live imaging, mineral oil (Sigma) was layered onto the Neurobasal medium in the dishes to prevent evaporation of the medium. The glass-bottom dishes were transferred to a temperature-controlled chamber (Bioptechs) on an Eclipse TE-2000E inverted microscope system (Nikon). BDNF $(100 \mathrm{ng} / \mathrm{ml})$ was added to the culture, and green and red fluorescent images were visualized using a $60 \times$ Plan-fluor objective on the TE-2000E microscope system equipped with $300 \mathrm{~W}$ xenon arc lamp and Lambda 10-3 multi-filter wheel system (Sutter Instruments). Time-lapse images were captured on a cooled EMCCD camera (Cascade:512B; Photometrics) using NIS-elements software (Nikon) at $10 \mathrm{~min}$ intervals for $60 \mathrm{~min}$ after addition of BDNF. Fluorescent images were analyzed using NIH Image J. Mean fluorescence intensities for EGFP and mCherry in growth cones were analyzed within individual growth cones as ROIs. Total fluorescence intensity of the ROI was then divided by area of the ROI to normalize for differences in growth cone area. The resulting total intensity/area was subtracted by a mean of two regions (background/area) near individual growth cones and then was defined as a normalized fluorescence intensity in a growth cone. The ratio of $\mathrm{gd} 2 \mathrm{G} / \mathrm{gd} 2 \mathrm{R}$ was calculated by ratio of the normalized fluorescence intensity of EGFP to that of mCherry. Each data point was calculated from at least eight growth cones, using a paired Student's $t$ test.

Xenopus embryo injection, spinal neuron culture, and growth cone turning assay. Blastomere injections of mRNA encoding ZBP1 wild-type (WT) or Y396F mutant into Xenopus embryo were performed as described previously (Wen et al., 2007). In brief, the mRNA of each construct was prepared using the mMESSAGE mMACHINE kit (Ambion), and 2-3 ng was microinjected into one blastomere of Xenopus embryos at the one- or two-cell stage together with 10 $\mathrm{mg} / \mathrm{ml}$ fixable FITC-dextran as the marker. Injected embryos were screened for their fluorescence and used later for cell cultures. Embryonic Xenopus spinal neurons isolated from stage 20-22 Xenopus embryos were cultured on glass coverslips coated with poly-D-lysine and laminin as described previously (Guirland et al., 2003; Wen et al., 2007). The cultures were kept at $20-22^{\circ} \mathrm{C}$ in a serum-free medium containing $50 \%$ (v/v) Leibovitz L-15 medium (Invitrogen), 50\% (v/v) Ringer's solution (in mM: $115 \mathrm{KCl}, 2 \mathrm{CaCl}_{2}, 2.6 \mathrm{KCl}$, and 10 HEPES, $\mathrm{pH} 7.4$ ), and $1 \%$ (wt/vol) BSA (Sigma-Aldrich). Growth cone turning induced by BDNF gradients was performed at room temperature according to the method described previously (Guirland et al., 2003). Microscopic gradients of chemicals were produced by the pipette application method described previously (Zheng et al., 1994). A standard pressure pulse of 3 pounds per square inch was applied to a glass pipette ( $1 \mu \mathrm{m}$ opening) at a frequency of $2 \mathrm{~Hz}$ with pulse duration of $20 \mathrm{~ms}$. The direction of growth cone extension at the beginning of the experiment was defined by the distal 20 $\mu \mathrm{m}$ segment of the neurite. The pipette tip was positioned $45^{\circ}$ from the initial direction of extension and $100 \mu \mathrm{m}$ away from the growth cone. We 
typically used a $20 \times$, numerical aperture 0.45 dry objective for microscopy and imaging during the turning assay. The digital images of the growth cone at the onset and end of the $30 \mathrm{~min}$ period were acquired by a CCD camera (C2400; Hamamatsu) and saved onto the hard drive. The images were then overlaid with pixel-to-pixel accuracy, and the trajectory of new neurite extension was traced using Photoshop (Adobe Systems). The turning angle was defined by the angle between the original direction of neurite extension and a line connecting the positions of the growth cone at the experiment onset and at the end of $30 \mathrm{~min}$ exposure to the gradient. Neurite extension was quantified by measuring the entire trajectory of net neurite growth over the $30 \mathrm{~min}$ period. Only growth cones extending $5 \mu \mathrm{m}$ or more were scored for turning responses. Turning angles were analyzed by the nonparametric MannWhitney test, because they do not follow a normal distribution.

\section{Results}

\section{Phosphorylation of ZBP1 in growth} cones in response to $\mathrm{BDNF}$

We investigated whether phosphorylation of ZBP1 at Tyr396 by Src provides a local mechanism to increase translation of $\beta$-actin mRNA. Src and ZBP1 colocalize at the cell periphery and beneath the membrane of non-neuronal cells (Hüttelmaier et al., 2005), but it is still unknown whether ZBP1 is phosphorylated by Src or SFKs at peripheral locations, such as in growth cones to regulate local translation in response to extracellular stimuli. To examine this, we prepared an anti-phospho-ZBP1 (Tyr396) antibody. The phosphorylation site is located just upstream of the $\mathrm{KH} 3$ and KH4 domains, which are essential for ZBP1 to bind to the zipcode sequence (Farina et al., 2003) (Fig. 1A). The neighboring amino acid sequence to Tyr396 is conserved among different species of ZBP1 but not in IMP2 and IMP3 homologs (Fig. $1 B$ ). ZBP1 is a member of a three gene family encoding $\mathrm{KH}$-domain containing mRNA-binding proteins, also termed IMP or VICKZ (Vg1RBP/ Vera, IMP-1,2,3, CRD-BP, KOC, ZBP1) proteins (Yisraeli, 2005). The phospho-antibody was shown to be ZBP1 specific as demonstrated by Western blot analysis of HEK293T cells expressing wild-type ZBP1 or IMP2/IMP3 homologs (supplemental Fig. S1, available at www.jneurosci.org as supplemental material). These data suggest that the phospho-antibody may recognize both the phosphor-tyrosine at 396 and the conserved surrounding sequence of ZBP1, which is not conserved between homologs (Fig. $1 B)$. In HEK293T cells coexpressing a wild-type or phospho-mutant (Y396F) form of ZBP1 with or without constitutively active Src, this antibody recognized wild-type ZBP1 phosphorylated by Src (Fig. $1 C$ ). Expression of the SFK Fyn, which is one of the neuronal SFKs, also resulted in a marked increase in levels of phospho-ZBP1 (Fig. 1D). An SFK-specific inhibitor, PP2 [4-amino-5-(4-chlorophenyl)7-(t-butyl)pyrazolo[3,4-d]pyrimidine], inhibited ZBP1 phosphorylation by Fyn (Fig. $1 D$ ). These results confirmed the specificity of the antibody to SFK-phosphorylated ZBP1 at Tyr396.

To examine whether BDNF induces the phosphorylation of endogenous ZBP1 in neurons, we applied BDNF to dissociated cortical neurons in culture and analyzed protein expression by Western blotting using the phospho-antibody to ZBP1. BDNF increased phosphorylation of ZBP1 at Tyr396 with a detectable peak at $15 \mathrm{~min}$ and decreased thereafter $(30 \mathrm{~min})$, although total levels of ZBP1 remained unchanged (Fig. 1E).
Next, we used the phospho-ZBP1 antibody in IF experiments to examine whether BDNF could stimulate local phosphorylation of ZBP1 in axons and growth cones. Anti-ZBP1 antibody was also used for double-labeled IF analysis to examine the total amount of ZBP1 in growth cones. BDNF increased phosphoZBP1 IF signals in growth cones at $10 \mathrm{~min}$ (Fig. 2A). Quantitative analysis demonstrated that both phospho-ZBP1 and total ZBP1 IF increased significantly, but the BDNF-induced increase in IF signal for phospho-ZBP1 (2.2 times) was greater than that observed for total ZBP1 (1.2 times) (Fig. $2 B$ ). As a result, the ratio of phospho-ZBP1 to total ZBP1 in growth cones increased 1.6 times after BDNF stimulation. These data indicate that BDNF not only increased ZBP1 accumulation in growth cones, as shown previously (Zhang et al., 2001), but also caused marked phosphorylation at its Tyr396. The present findings support the proposed model that local phosphorylation of ZBP1 provides a switch to activate local $\beta$-actin mRNA translation (Dahm and Kiebler, 2005).

\section{ZBP1 Y396F phospho-mutant has no effect on ZBP1 and $\boldsymbol{\beta}$-actin mRNA localization in growth cones}

Previous work has shown that the nonphosphorylatable mutant of ZBP1, Y396F (Tyr at 396 is converted to Ala: Y396F), has dramatically increased affinity for $\beta$-actin mRNA (Hüttelmaier et al., 2005). Thus, because Y396F remains tightly bound $\beta$-actin mRNA and cannot be phosphorylated to release the $\beta$-actin mRNA, it could act in a dominant-negative manner to impair the local synthesis of $\beta$-actin. However, the effects of Y396F on the regulation of local $\beta$-actin synthesis and cell motility have not been studied. We hypothesized that expression of the nonphosphorylatable mutant Y396F would inhibit BDNF regulation of local $\beta$-actin synthesis and growth cone motility. As a first step to test this model, we investigated whether Y396F affects ZBP1 and/or $\beta$-actin mRNA localization. The hypothesis predicts that Y396F would not impair mRNA or ZBP1 localization yet prevents the activation of local translation. Wild-type or Y396F mutant forms of flag-tagged mCherry-ZBP1 (ZBP1 WT or Y396F) were cotransfected into cortical neurons with GFP as a morphologic marker. The tagged ZBP1 was visualized through the use of 
A
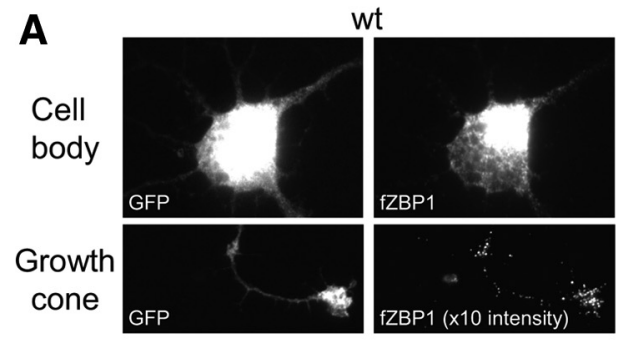

B
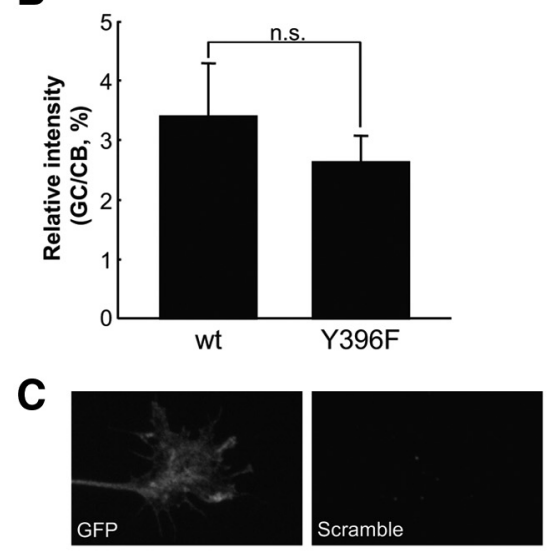

D
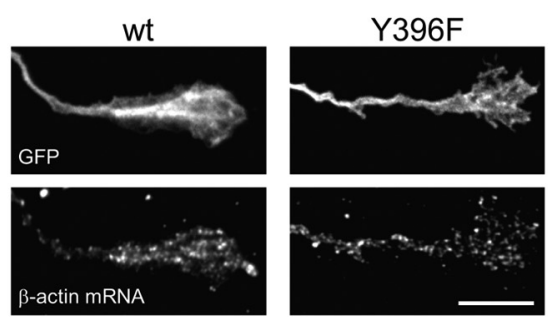

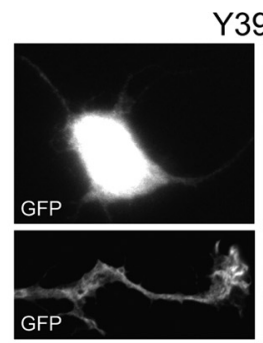

Y396F
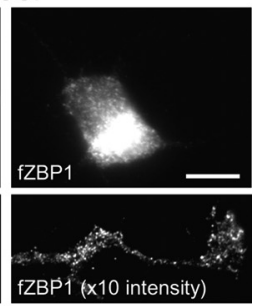

amined the effect of the Y396F mutant on the localization of endogenous $\beta$-actin mRNA in growth cones using FISH with digoxigenin-labeled antisense probes. As shown previously (Bassell et al., 1998), antisense probes for $\beta$-actin mRNA were visualized as punctate signals in growth cones, but scrambled probes did not detect appreciable signals (Fig. 3C). Quantitative analysis showed that FISH signals of $\beta$-actin mRNA in growth cones of neurons transfected with the Y396F mutant were not significantly different from those transfected with ZBP1 WT (Fig. 3D,E). These data suggest that phosphorylation of ZBP1 at Tyr396 is not necessary for the localization of ZBP1 and $\beta$-actin mRNA to growth cones.

\section{ZBP1 Y396F phospho-mutant} suppresses the BDNF-induced increase in $\boldsymbol{\beta}$-actin in growth cones, which is normally protein synthesis dependent Next, we examined the role of tyrosine phosphorylation of ZBP1 in local $\beta$-actin synthesis in growth cones. The phosphomutant Y396F was shown previously to strongly repress $\beta$-actin translation in HEK293 cells as analyzed using luciferase reporters (Hüttelmaier et al., 2005). Thus, we investigated whether the Y396F mutant would block BDNF-induced synthesis of endogenous $\beta$-actin in neurons, suggesting an inability to derepress mRNA translation. It has yet to be examined whether this mutant impairs the regulation of $\beta$-actin synthesis and localization. Cortical neurons transfected with ZBP1 WT or Y396F were stimulated with BDNF, and endogenous $\beta$-actin protein localization in growth cones was analyzed by IF using an anti- $\beta$ actin antibody. BDNF promoted an increase in $\beta$-actin protein in growth cones of neurons transfected with wild-type but not the Y396F mutant (Fig. 4A). The increase in $\beta$-actin protein was more prominent in filopodia and lamellipodia of growth cones. Quantitative analysis demonstrated that the increase in $\beta$-actin in growth cones of neurons transfected with ZBP1 WT, but not Y396F, was significant an anti-flag antibody. ZBP1 WT and Y396F were similarly localized in growth cones (Fig. 3A). ZBP1 WT and Y396F were also similarly localized in peripheral leading edges of feline kidney cortex epithelial CrFK cells (supplemental Fig. S3, available at www.jneurosci.org as supplemental material). To examine localization of ZBP1 WT and Y396F in growth cones quantitatively, we analyzed the ratio of exogenously expressed ZBP1 in growth cones to cell bodies using an anti-flag antibody. Using a normalized ratio for the flag-tagged ZBP1 expression, we found that levels of ZBP1 Y396F in growth cones were similar to ZBP1 WT (Fig. $3 B$ ), indicating no significant difference in the localization of ZBP1 between wild-type and phospho-mutant. We then ex-

at 15 and $30 \mathrm{~min}$ after BDNF stimulation (Fig. $4 B$ ). In growth cones of neurons transfected with wild-type ZBP1, the increase in $\beta$-actin was blocked by a protein synthesis inhibitor, anisomycin (Fig. 4C). These data suggest that BDNF facilitates $\beta$-actin localization in growth cones in a protein synthesis-dependent manner via the phosphorylation of ZBP1 at Tyr396.

\section{BDNF stimulates local translation of a $\boldsymbol{\beta}$-actin translation reporter in growth cones in an SFK-dependent manner} To examine the role of tyrosine phosphorylation in the regulation of local protein synthesis in growth cones, we developed a modified method to normalize and quantify local translation in a 
growth cone by cotransfection of a GFP$\beta$-actin $3^{\prime}$ UTR translation reporter and an mCherry reporter for normalization (Fig. 5A). We created two versions of the GFP translation reporter, either with or without the full length of $3^{\prime}$ UTR of $\beta$-actin. We used a membrane-tagged, short-lived EGFP variant, as used previously with the calcium/calmodulindependent kinase II $\alpha$ (CaMKII $\alpha)$ 3' UTR as a translation reporter in dendrites (Aakalu et al., 2001). We confirmed that the $\beta$-actin version of the GFP reporter (gd2G + 3' UTR) (Fig. 5A) demonstrated increased fluorescence intensity over time in a protein synthesis-dependent manner (supplemental Figs. S4, S5, available at www.jneurosci.org as supplemental material), similar to the CaMKII $\alpha$ 3' UTR reporter in dendrites (Aakalu et al., 2001). Normalization of EGFP intensity to mCherry was used to quantify translational responses of individual growth cones in response to BDNF that were mediated by the $3^{\prime}$ UTR of $\beta$-actin (Fig. $5 A, B)$. Because the mCherry reporter (gd2R) has the same membrane-tagged and short-lived sequences of the EGFP reporter (gd2G + 3' UTR) but lacks the $3^{\prime}$ UTR, the turnover and membrane localization of the mCherry reporter should be the same as the GFP reporter. This allowed for normalization of GFP fluorescence caused by variability in thickness of growth cones and diffusion of the GFP reporter during live imaging for $1 \mathrm{~h}$. A limitation using single fluorescent reporters for analysis of local translation is that one cannot eliminate the possibility that some of the GFP signal was derived from transport or diffusion from the neurite into the growth cones; therefore, the normalization of mCherry signal within the same cells provides an ideal internal control.

As an additional means to spatially resolve local translation within an individual growth cone in a manner distinguishable from translation in the cell body and subsequent diffusion into the axon, the analysis described used a novel hanging drop neuron ball culture method that permits analysis of growth cones from axons that are $>1 \mathrm{~mm}$ in length (supplemental Fig. S6, available at www.jneurosci.org as supplemental material) (see Materials and Methods). Because membrane-tagged proteins such as the GFP and mCherry reporters diffuse at $50 \mu \mathrm{m} / \mathrm{h}$, we could rule out the contribution of protein synthesis in cell bodies as a means to change the accumulation of GFP in growth cones relative to normalization with mCherry in response to short-term stimulation with BDNF.

Using this normalization approach and analysis of growth cones from axons $>1 \mathrm{~mm}$ in length, the GFP reporter with the $\beta$-actin 3' UTR (gd2G $+3^{\prime}$ UTR) showed an increase in fluorescence intensity in response to stimulation (Fig. $5 B, C$ ). When BDNF was applied to a neuron ball culture expressing the $\mathrm{gd} 2 \mathrm{G}+3^{\prime}$ UTR and mCherry reporters, the GFP, but not mCherry, fluorescence increased in the axon and growth cone at $60 \mathrm{~min}$ (Fig. 5B). We used a pseudocolor intensity map with the GFP reporter to illustrate how the fluorescence intensity increased in the palm of a growth cone after BNDF stimulation (Fig. 5B). To normalize the GFP signal, we calculated GFP/mCherry fluorescence ratios in growth cones. BDNF stimulation increased the fluorescence ratio after $30 \mathrm{~min}$, and the ratio reached a $20 \%$ increase in growth cones at $60 \mathrm{~min}$ (Fig. $5 \mathrm{C}$ ). When the GFP reporter without the $3^{\prime}$ UTR of $\beta$-actin was cotransfected with the mCherry reporter, BDNF did not change the fluorescence ratio (Fig. 5C). These data indicate that the BDNF-induced increase in the fluorescence ratio is $3^{\prime}$ UTR dependent. Next, we applied the SFK inhibitor PP2 to this assay system. PP2, but not its inactive derivative form PP3 (4-amino-7-phenylpyrazol[3,4- $d]$ pyrimidine), suppressed the BDNF-induced increase in the fluorescence ratio in growth cones cotransfected with gd2G $+3^{\prime}$ UTR and the mCherry reporter (Fig. 5D). These data indicate a role for SFK in local protein synthesis mediated by the $3^{\prime}$ UTR of $\beta$-actin. These data are consistent with our finding that Y396F blocks the protein synthesis-dependent increase in $\beta$-actin in growth cones in response to BDNF and suggest that BDNF promotes local protein synthesis of $\beta$-actin in growth cones mediated by phosphorylation of ZBP1 at Tyr396 by SFKs.

\section{Tyrosine phosphorylation of ZBP1 at 396 is necessary for growth cone turning toward a BDNF gradient}

To examine the role of tyrosine phosphorylation of ZBP1 in growth cone turning, we used an in vitro turning assay. In this assay, cultured embryonic Xenopus laevis spinal neurons transfected with WT or the tyrosine mutant of GFP-ZBP1, Y396F, were exposed to an extracellular gradient of BDNF (Fig. 6). Xenopus neurons have been used extensively in growth cone turning assays (Campbell and Holt, 2001; Leung et al., 2006; Yao et al., 2006). Both GFP-ZBP1 WT and Y396F were expressed in cell bodies, axons, and growth cones (Fig. $6 A$, inset), as visualized by GFP fluorescence. A gradient created by pulsatile ejection of 
A

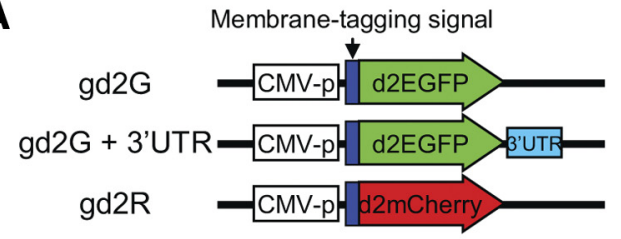

B
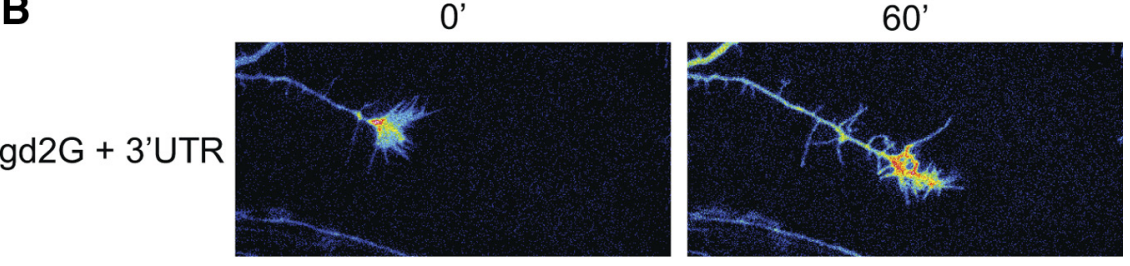

$\operatorname{gd} 2 \mathrm{R}$
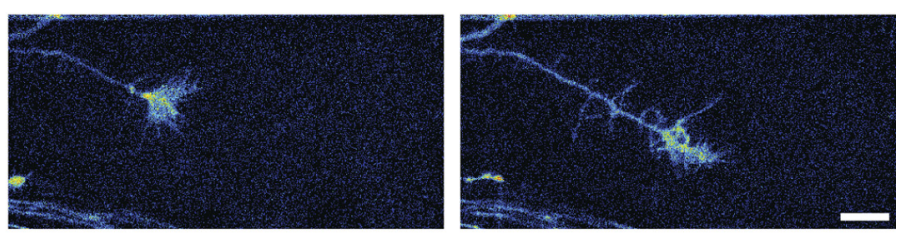

C

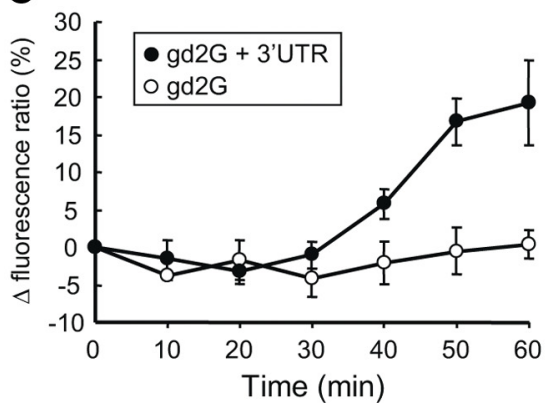

D

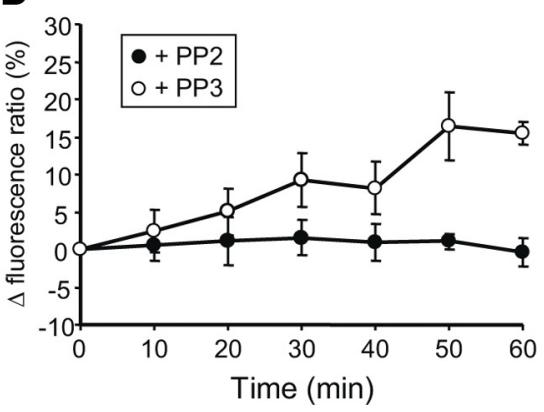

Figure 5. The Src family kinase inhibitor PP2 suppressed BDNF-induced local translation in growth cones using a GFP reporter with the $3^{\prime}$ UTR of $\beta$-actin. Neurons were transfected with designated reporters and cultured as neuron balls extending axons $>1$ $\mathrm{mm}$ in length (see Materials and Methods) (supplemental Fig. S6, available at www.jneurosci.org as supplemental material). $\boldsymbol{A}$, Vector structures of fluorescent reporters. Both GFP and mCherry reporters were fused with a membrane-targeting sequence (dual palmitoylation from GAP43) and a sequence for shorter half-life (d2EGFP and d2mCherry). The GFP reporter (gd2G) contains the 3' UTR sequence of rat $\beta$-actin, but the mCherry reporter ( $g d 2 R)$ does not contain this sequence. CMV, Cytomegalovirus. $\boldsymbol{B}$, Representative images of growth cones of cortical neurons in neuron ball cultures cotransfected with gd2G $+3^{\prime}$ UTR and gd2R. Pseudocolored images show that the absence of fluorescent signal is indicated by black, with increasing fluorescence indicated by transitions to blue, green, yellow, and red. Note that BDNF stimulation increased the fluorescence of $g d 2 G+3^{\prime}$ UTR, but not gd2R, after $60 \mathrm{~min}$. Scale bar, $10 \mu \mathrm{m}$. C, Effect of the $\beta$-actin 3' UTR on expression of the GFP reporter in growth cones. The GFP reporter ( $\mathrm{gd} 2 \mathrm{G}$ or gd2G + 3' UTR) was cotransfected with gd2R into cortical neurons. Neurons were stimulated with BDNF, and fluorescence ratios of the GFP reporters to $\mathrm{gd} 2 \mathrm{R}$ were calculated at indicated times and then normalized to the initial fluorescence ratio. The fluorescence ratio of $\mathrm{gd} 2 \mathrm{G}+3^{\prime}$ UTR to $\mathrm{gd} 2 \mathrm{R}$ increased from $30 \mathrm{~min}$ after BDNF stimulation, but the fluorescence ratio of $\mathrm{gd} 2 \mathrm{G}$ did not. $D$, The SFK inhibitor PP2, but not its inactive derivative PP3, inhibited the increase of the fluorescence ratio of gd2G $+3^{\prime}$ UTR to gd2R after BDNF stimulation.

BDNF $(5 \mu \mathrm{g} / \mathrm{ml}$ in pipette) induced attractive turning of the growth cone of the ZBP1 WT neuron toward the pipette (Fig. $6 A)$. The traces of individual growth cones demonstrate that the majority of growth cones turned toward the BDNF pipette (Fig. $6 A)$. The average turning angle of growth cones expressing ZBP1 WT was $18.4 \pm 5.0^{\circ}$ (mean \pm SEM) (Fig. $6 B$ ). This turning angle was comparable with nontransfected control neurons (data not shown), suggesting that overexpression of ZBP1 WT has no effect on growth cone turning. In contrast, BDNF-induced attraction was completely abolished in neurons expressing ZBP1 Y396F (Fig. 6A, $B$ ). However, axon extension was not affected in neurons expressing ZBP1 WT or Y396F (Fig. 6C). These data indicate that ZBP1 Y396F has a dominant-negative effect on growth cone turning toward BDNF, similar to its impairment of the protein synthesis- dependent increase in endogenous $\beta$-actin protein content within growth cones (Fig. 4). Together, this suggests that tyrosine phosphorylation of ZBP1 at 396 has a critical role in local translation of $\beta$-actin and growth cone turning.

\section{Discussion}

Phosphorylation of zipcode binding protein is required for local $\boldsymbol{\beta}$-actin synthesis in growth cones

Our findings indicate that local phosphorylation of ZBP1 by SFK regulates the local translation of $\beta$-actin and growth cone turning in response to BDNF stimulation. Previous work has shown that ZBP1 binding to the $3^{\prime}$ UTR represses translation of $\beta$-actin mRNA, whereas phosphorylation of ZBP1 at Tyr396 by Src decreases its affinity for $\beta$-actin mRNA (Hüttelmaier et al., 2005). Furthermore, fluorescence resonance energy transfer analysis depicted a close association between Src and ZBP1 near the membrane (Hüttelmaier et al., 2005). These data suggest the proposed model whereby physiological signals may lead to an increase in ZBP1 phosphorylation at Tyr396 at the cell periphery that then activates local $\beta$-actin mRNA translation. However, no previous evidence has yet directly demonstrated that ZBP1 is phosphorylated to promote $\beta$-actin translation locally.

Here we present three lines of evidence in support of this model. First, we show that endogenous levels of phosphorylated ZBP1 are rapidly increased within growth cones during BDNF stimulation for 10 min (Fig. 2). Second, we observed that overexpression of the nonphosphorylatable ZBP1 mutant, Y396F, inhibited the accumulation of endogenous $\beta$-actin within growth cones, a response that normally depends on protein synthesis to reach maximal expression (Fig. 4). It is important to note that, although the enrichment of $\beta$-actin protein is impaired by $\mathrm{Y} 396 \mathrm{~F}$, there is no adverse effect of this phospho-mutant on the localization of ZBP1 and/or $\beta$-actin mRNA (Fig. 3). These data suggest that the mutation at Tyr396 acts as a dominant-negative mutation for translation but not localization of ZBP1 or $\beta$-actin mRNA. Last, we show that local translation of a GFP reporter with the $3^{\prime}$ UTR of $\beta$-actin is induced by BDNF and requires Src activity (Fig. 5). Together, these data are consistent with a model that BDNF induces the Src-dependent phosphorylation of ZBP1, which is necessary for local $\beta$-actin mRNA translation. Because SFKs share redundant roles in regulation of phosphorylation in neurons (Arnaud et al., 2003), we consider that other SFKs such as Fyn could likely also phosphorylate ZBP1 in growth cones. From the present data, we note that transient increases in the tyrosine phosphorylation of ZBP1 and subsequent $\beta$-actin translation, as measured by IF and the fluorescent 
reporters, occur more strongly in growth cones than in peripheral axons after BDNF stimulation (Figs. 2, 4, 5). These data suggest that the ZBP1 phosphorylation and $\beta$-actin translation are regulated spatially and temporally as in the proposed model. Because the phosphorylation of Tyr396 reduces binding of ZBP1 to $\beta$-actin mRNA (Hüttelmaier et al., 2005), the spatial and temporal regulation of ZBP1 phosphorylation may provide a switch to activate translation. However, additional work is needed to clarify the molecular mechanism of translational derepression in response to phosphorylation by Src. It was reported that ZBP1 interferes directly with $80 \mathrm{~S}$ ribosome assembly by blocking 60S joining (Hüttelmaier et al., 2005). Therefore, phosphorylation of ZBP1 would release its binding from the mRNA and remove this negative inhibition. Another possible mechanism is that phosphorylation of ZBP1 may modify its interaction with other components of the messenger ribonucleoprotein complex that are repressed within RNA granules (Kiebler and Bassell, 2006). Additional work is necessary to clarify the precise mechanism of ZBP1-mediated translational control in growth cones.

\section{Phosphorylation of mRNA-binding proteins required for axon extension and/or guidance}

There is an increasing list of mRNAbinding proteins localized in axons and growth cones (Kiebler and Bassell, 2006), but it has been unclear which are required to regulate local protein synthesis that is necessary for axon guidance. Regulation of CPEB-mediated initiation of translation by phosphorylation has been intensely studied in several systems and is implicated in protein synthesis in dendrites and at synapses (Richter, 2007). Moreover, the role of CPEB in axonal development was shown recently, too. Whereas CPEB1 knockdown in Xenopus embryos did not result in axon guidance defects, expression of cDNA coding a nonphosphorylatable mutant (S147A, S180A) of CPEB1, which cannot undergo the phosphorylation critical for activating the translation of its target mRNA, suppressed retinal axon extension in vivo (Lin et al., 2009). This result suggests that phosphorylation of CPEB1 regulates axon extension via local translation, although it remains unclear which target mRNAs are necessary for axon extension. The role of FMRP in the regulation of protein synthesis in dendrites and synapses has also been extensively studied (Bassell and Warren, 2008). The phosphorylation status of FMRP appears to play a role in the reversible regulation of mRNA translation (Narayanan et al., 2007, 2008). FMRP may similarly regulate axonal protein synthesis. FMRP is localized in granules within axons and growth cones of cultured neurons (Antar et al., 2006; Hengst et al., 2006) and in axons in vivo (Price et al., 2006; Murashov et al., 2007). Also, Fmr1 knock-out (KO) neurons showed reduced growth cone motility (Antar et al., 2006). More recently, growth cones of
Fmr1 KO hippocampal neurons were shown to lack the protein synthesis-dependent collapse response induced by Sema3A, a repulsive axon guidance factor ( $\mathrm{Li}$ et al., 2009). Furthermore, axonal projections from layer 4 to 3 in the cortex were improperly regulated and defasciculated in Fmr1 KO neurons in vivo (Bureau et al., 2008). These findings suggest that FMRP plays an important role in axon guidance, yet it remains unclear whether this involves FMRP-mediated local mRNA translation. Because the FMRP target mRNA encoding the microtubule associated protein $1 \mathrm{~B}$ is localized to axonal growth cones ( $\mathrm{Li}$ et al., 2009), this may be a probable candidate. Future work is needed to assess how axon guidance factors may regulate FMRP phosphorylation status and local mRNA translation.

In this study, ZBP1 is identified as an essential factor whose phosphorylation status can be locally regulated to activate local protein synthesis necessary for growth cone turning. Previous studies have demonstrated that BDNF and netrin-1 can promote the asymmetric localization of $\beta$-actin mRNA and VgRBP (Xenopus ZBP1 homolog) in growth cones and the local protein synthesis of $\beta$-actin mRNA in growth cones (Leung et al., 2006; Yao et al., 2006). AMOs against the zipcode to interfere with the interaction between ZBP1 and $\beta$-actin mRNA suppressed attrac- 
tive growth cone turning toward a source of BDNF (Yao et al., 2006). In this report, we demonstrated that a nonphosphorylatable mutant form of ZBP1, Y396F, which is a dominant-negative mutant for local translation, attenuated attractive turning of growth cones to a BDNF source. This is the first direct evidence to show that $\mathrm{ZBP} 1$ is required for growth cone turning. A possible mechanism may be that SFK activated by focal stimulation of BDNF phosphorylates ZBP1 in growth cones, which results in the release of $\beta$-actin mRNA and allows for local translation. Locally synthesized $\beta$-actin toward an attractive source would then be actively incorporated into polymerizing actin filaments on the stimulated side of the growth cone. This asymmetrically localized $\beta$-actin, derived by local translation, may bias growth cone turning to the attractive cue. These data are consistent with previous observations that focal stimulation of growth cones by BDNF promotes not only the asymmetric localization of $\beta$-actin in growth cones in a protein synthesis-dependent manner but also an increase in F-actin on the near side (Leung et al., 2006; Yao et al., 2006).

Additional work is needed to identify the precise kinetics and sites of local $\beta$-actin mRNA translation and $\beta$-actin protein accumulation in growth cones. Using fluorescent reporters, BDNF induced the increase in normalized fluorescence intensity in growth cones after 30-60 min in a 3' UTR-dependent manner. However, the increase in endogenous $\beta$-actin staining in growth cones (15-30 $\mathrm{min})$ preceded the increase in fluorescence intensity using the GFP/ $\beta$-actin 3' UTR reporter (after $30 \mathrm{~min}$ ). The delay in the expression of local fluorescence intensity using the GFP reporter is likely attributable to the slow maturation of GFP (half-time is $\sim 60 \mathrm{~min}$ ); thus, it is probable that the actual $\beta$-actin synthesis in growth cones may start within 15 min after stimulation. Immunostaining analysis revealed that the increase in $\beta$-actin in growth cones mainly occurred in filopodia and lamellipodia after BDNF stimulation. However, the fluorescent signal of the $\beta$-actin reporter is distributed intensely in the central part of growth cones. One possible explanation for this discrepancy of localization is that newly synthesized $\beta$-actin in the central domain of growth cones is incorporated preferentially into actively polymerizing actin filaments in filopodia and lamellipodia after its synthesis more proximally. This possibility is supported by the fact that phosphorylated ZBP1 is mainly localized in the central part of growth cones. Furthermore, it is reported that $\beta$-actin mRNA is bound to microtubules in the growth cone palm, the location where polyribosomes are detected in the growth cone (Bassell et al., 1998). ZBP1 and $\beta$-actin mRNA colocalize in the growth cone central domain (Zhang et al., 2001; Yao et al., 2006). These findings suggest that the major sites of asymmetric protein synthesis of $\beta$-actin are in the central domain of growth cones. However, some ZBP1/VgRBP target directly into filopodia extending toward a gradient source (Leung et al., 2006), suggesting the possibility that some translation may initiate directly within filopodia. Nonetheless, asymmetric $\beta$-actin synthesis within the central domain may be sufficient to establish a gradient in locally synthesized monomers that can be readily incorporated into F-actin within filopodia on the near side of the growth cone.

Future studies are needed to identify which axon guidance cues promote phosphorylation of ZBP1 in vivo and to analyze possible axon guidance defects in vivo using overexpression of the dominant-negative form of ZBP1 and/or loss of function of ZBP1. Our findings for a role of ZBP1 regulation in local protein synthesis and growth cone turning also provide motivation to study where and when ZBP1 regulation and $\beta$-actin mRNA translation occur during other aspects of neuronal development, such as synaptogenesis, formation of neural circuits, and regeneration after nerve injury. We suggest that ZBP1 has a critical role in developmental and pathophysiological regulation of local translation of $\beta$-actin mRNA, which may have an impact on growth cone dynamics to extend axons and wire precise neural circuits.

\section{References}

Aakalu G, Smith WB, Nguyen N, Jiang C, Schuman EM (2001) Dynamic visualization of local protein synthesis in hippocampal neurons. Neuron 30:489-502.

Antar LN, Li C, Zhang H, Carroll RC, Bassell GJ (2006) Local functions for FMRP in axon growth cone motility and activity-dependent regulation of filopodia and spine synapses. Mol Cell Neurosci 32:37-48.

Arnaud L, Ballif BA, Förster E, Cooper JA (2003) Fyn tyrosine kinase is a critical regulator of disabled-1 during brain development. Curr Biol 13:9-17.

Bassell GJ, Kelic S (2004) Binding proteins for mRNA localization and local translation, and their dysfunction in genetic neurological disease. Curr Opin Neurobiol 14:574-581.

Bassell GJ, Warren ST (2008) Fragile X syndrome: loss of local mRNA regulation alters synaptic development and function. Neuron 60:201-214.

Bassell GJ, Zhang H, Byrd AL, Femino AM, Singer RH, Taneja KL, Lifshitz LM, Herman IM, Kosik KS (1998) Sorting of $\beta$-actin mRNA and protein to neurites and growth cones in culture. J Neurosci 18:251-265.

Bramham CR, Wells DG (2007) Dendritic mRNA: transport, translation and function. Nat Rev Neurosci 8:776-789.

Bureau I, Shepherd GM, Svoboda K (2008) Circuit and plasticity defects in the developing somatosensory cortex of FMR1 knock-out mice. J Neurosci 28:5178-5188.

Campbell DS, Holt CE (2001) Chemotropic responses of retinal growth cones mediated by rapid local protein synthesis and degradation. Neuron 32:1013-1026.

Dahm R, Kiebler M (2005) Cell biology: silenced RNA on the move. Nature 438:432-435.

Eom T, Antar LN, Singer RH, Bassell GJ (2003) Localization of a $\beta$-actin messenger ribonucleoprotein complex with zipcode-binding protein modulates the density of dendritic filopodia and filopodial synapses. J Neurosci 23:10433-10444.

Farina KL, Huttelmaier S, Musunuru K, Darnell R, Singer RH (2003) Two ZBP1 KH domains facilitate beta-actin mRNA localization, granule formation, and cytoskeletal attachment. J Cell Biol 160:77-87.

Goslin K, Asmussen H, Banker G (1998) Rat hippocampal neurons in low density culture. In: Culturing nerve cells, Ed 2 (Banker G, Golsin K, eds), pp 339-371. Cambridge, MA: Massachusetts Institute of Technology.

Guirland C, Buck KB, Gibney JA, DiCicco-Bloom E, Zheng JQ (2003) Direct cAMP signaling through G-protein-coupled receptors mediates growth cone attraction induced by pituitary adenylate cyclase-activating polypeptide. J Neurosci 23:2274-2283.

Hengst U, Cox LJ, Macosko EZ, Jaffrey SR (2006) Functional and selective RNA interference in developing axons and growth cones. J Neurosci 26:5727-5732.

Hüttelmaier S, Zenklusen D, Lederer M, Dictenberg J, Lorenz M, Meng X, Bassell GJ, Condeelis J, Singer RH (2005) Spatial regulation of betaactin translation by Src-dependent phosphorylation of ZBP1. Nature 438:512-515.

Kiebler MA, Bassell GJ (2006) Neuronal RNA granules: movers and makers. Neuron 51:685-690.

Leung KM, van Horck FP, Lin AC, Allison R, Standart N, Holt CE (2006) Asymmetrical beta-actin mRNA translation in growth cones mediates attractive turning to netrin-1. Nat Neurosci 9:1247-1256.

Li C, Bassell GJ, Sasaki Y (2009) Fragile X mental retardation protein is involved in protein synthesis-dependent collapse of growth cones induced by Semaphorin-3A. Front Neural Circuits 3:11.

Lin AC, Holt CE (2007) Local translation and directional steering in axons. EMBO J 26:3729-3736.

Lin AC, Tan CL, Lin CL, Strochlic L, Huang YS, Richter JD, Holt CE (2009) Cytoplasmic polyadenylation and cytoplasmic polyadenylation elementdependent mRNA regulation are involved in Xenopus retinal axon development. Neural Dev 4:8.

Martin KC, Ephrussi A (2009) mRNA localization: gene expression in the spatial dimension. Cell 136:719-730. 
Murashov AK, Chintalgattu V, Islamov RR, Lever TE, Pak ES, Sierpinski PL, Katwa LC, Van Scott MR (2007) RNAi pathway is functional in peripheral nerve axons. FASEB J 21:656-670.

Narayanan U, Nalavadi V, Nakamoto M, Pallas DC, Ceman S, Bassell GJ, Warren ST (2007) FMRP phosphorylation reveals an immediate-early signaling pathway triggered by group I mGluR and mediated by PP2A. J Neurosci 27:14349-14357.

Narayanan U, Nalavadi V, Nakamoto M, Thomas G, Ceman S, Bassell GJ, Warren ST (2008) S6K1 phosphorylates and regulates fragile X mental retardation protein (FMRP) with the neuronal protein synthesisdependent mammalian target of rapamycin (mTOR) signaling cascade. J Biol Chem 283:18478-18482.

Nielsen J, Christiansen J, Lykke-Andersen J, Johnsen AH, Wewer UM, Nielsen FC (1999) A family of insulin-like growth factor II mRNAbinding proteins represses translation in late development. Mol Cell Biol 19:1262-1270.

Piper M, Holt C (2004) RNA translation in axons. Annu Rev Cell Dev Biol 20:505-523.

Price TJ, Flores CM, Cervero F, Hargreaves KM (2006) The RNA binding and transport proteins staufen and fragile $\mathrm{X}$ mental retardation protein are expressed by rat primary afferent neurons and localize to peripheral and central axons. Neuroscience 141:2107-2116.

Richter JD (2007) CPEB: a life in translation. Trends Biochem Sci 32: $279-285$.

Ross AF, Oleynikov Y, Kislauskis EH, Taneja KL, Singer RH (1997) Characterization of a beta-actin mRNA zipcode-binding protein. Mol Cell Biol 17:2158-2165.

Santangelo PJ, Lifland AW, Curt P, Sasaki Y, Bassell GJ, Lindquist ME, Crowe
JE Jr (2009) Single molecule-sensitive probes for imaging RNA in live cells. Nat Methods 6:347-349.

Sasaki Y, Cheng C, Uchida Y, Nakajima O, Ohshima T, Yagi T, Taniguchi M, Nakayama T, Kishida R, Kudo Y, Ohno S, Nakamura F, Goshima Y (2002) Fyn and Cdk5 mediate semaphorin-3A signaling, which is involved in regulation of dendrite orientation in cerebral cortex. Neuron 35:907-920.

Steward O, Schuman EM (2001) Protein synthesis at synaptic sites on dendrites. Annu Rev Neurosci 24:299-325.

Sutton MA, Schuman EM (2006) Dendritic protein synthesis, synaptic plasticity, and memory. Cell 127:49-58.

Tiruchinapalli DM, Oleynikov Y, Kelic S, Shenoy SM, Hartley A, Stanton PK, Singer RH, Bassell GJ (2003) Activity-dependent trafficking and dynamic localization of zipcode binding protein 1 and $\beta$-actin mRNA in dendrites and spines of hippocampal neurons. J Neurosci 23:3251-3261.

Wen Z, Han L, Bamburg JR, Shim S, Ming GL, Zheng JQ (2007) BMP gradients steer nerve growth cones by a balancing act of LIM kinase and Slingshot phosphatase on ADF/cofilin. J Cell Biol 178:107-119.

Yao J, Sasaki Y, Wen Z, Bassell GJ, Zheng JQ (2006) An essential role for beta-actin mRNA localization and translation in $\mathrm{Ca}^{2+}$-dependent growth cone guidance. Nat Neurosci 9:1265-1273.

Yisraeli JK (2005) VICKZ proteins: a multi-talented family of regulatory RNA-binding proteins. Biol Cell 97:87-96.

Zhang HL, Eom T, Oleynikov Y, Shenoy SM, Liebelt DA, Dictenberg JB, Singer RH, Bassell GJ (2001) Neurotrophin-induced transport of a betaactin mRNP complex increases beta-actin levels and stimulates growth cone motility. Neuron 31:261-275.

Zheng JQ, Felder M, Connor JA, Poo MM (1994) Turning of nerve growth cones induced by neurotransmitters. Nature 368:140-144. 